\title{
Vascular Endothelial Growth Factor (VEGF) Serum Concentration Changes during Chemotherapy in Patients with Lung Cancer
}

\author{
YASUKO KIDO \\ Department of Medicine, Kurume University School of Medicine, \\ Kurume 830-0011, Japan
}

\begin{abstract}
Summary: Vascular endothelial growth factor (VEGF) is one of the most important angiogenic factors. This study aimed at clarifying the clinical significance of the changes in the serum VEGF (S-VEGF) concentrations in patients with lung cancer during anticancer chemotherapy. Subjects comprised 29 patients with lung cancer (13 adenocarcinomas, 7 squamous cell carcinomas, and 9 small cell carcinomas) who were treated with cisplatin-based chemotherapy. S-VEGF was measured by ELISA. We compared the S-VEGF concentrations between the responders and nonresponders to anticancer chemotherapy. S-VEGF concentrations before treatment of the chemotherapy (pretreatment S-VEGF concentrations) were correlated with the number of WBC, neutrophil count, monocyte count and platelet count but not the lymphocyte count. The mean pretreatment S-VEGF concentrations in responders and those in nonresponders were not significantly different, $509.7 \mathrm{pg} / \mathrm{ml}$ in the former and $382.8 \mathrm{pg} / \mathrm{ml}$ in the latter, respectively. The SVEGF concentrations in the responders decreased to a mean of $356.0 \mathrm{pg} / \mathrm{ml}$ and $304.1 \mathrm{pg} / \mathrm{ml}$ during and at the end of therapy, respectively while those in the nonresponders increased to a mean of $474.2 \mathrm{pg} / \mathrm{ml}$ and $598.4 \mathrm{pg} / \mathrm{ml}$ during and at the end of therapy. The S-VEGF concentration changes in the responders were significantly different from those in the nonresponders $(p=.006)$. The S-VEGF concentration may relate to tumor burden, however it may not be a good marker for tumor burden, because it can be influenced by various factors such as neutrophil which increases during infection. A decrease in S-VEGF concentrations may improve neoangiogenesis and the immune response, and may correlate with improvements in the quality of life and survival of patients.
\end{abstract}

Key words VEGF, chemotherapy, lung cancer, prognosis, response

\section{INTRODUCTION}

Various cytokines and several tumor growth factors seem to stimulate cancer cell proliferation and dissemination at least in part by inducing vascular endothelial growth factor (VEGF) secretion [1]. Many types of cells produce these cytokines and growth factors in patients with cancer, including lung cancer. VEGF has several biological effects that can induce the neoangiogenic process, and the induction of angiogenesis seems to be the most important event responsible for tumor invasion and dissemination [2].
As a result, VEGF undoubtedly supports the enlargement of solid tumors. However, few studies have examined the relationship between response to chemotherapy and the serum VEGF (S-VEGF) concentrations in patients with lung cancer. The present study investigated the changes in the SVEGF concentration of patients with lung cancer during anticancer chemotherapy. We wanted to clarify the clinical significance of any changes in the SVEGF concentration. In addition, we wanted to clarify the association of the S-VEGF concentrations with the blood cell count, including neutrophils, 
lymphocytes, monocytes, and platelets, as well as the CRP.

\section{PATIENTS AND METHODS}

The S-VEGF concentrations were measured during anticancer chemotherapy in 29 patients with histologically proven lung cancer. These patients were diagnosed and treated in the Department of Internal Medicine, Kurume University School of Medicine, between 1999 and 2000. Informed consent was obtained from the patients who participated in this trial. All patients were treated with cisplatinbased chemotherapy. The clinical response was evaluated according to the WHO criteria. The clinical response consisted of a partial response (PR) in 11/29 (38\%) patients, a complete response (CR) in 1/29 $(3 \%)$, stable disease (SD) in $5 / 29(17 \%)$, and progressive disease (PD) in 12/29 (41\%). The responders included 12 patients who achieved a CR or PR. The nonresponders included 17 patients with SD or PD (Table 1).

To evaluate S-VEGF secretion, venous blood samples were collected in the morning before the start of chemotherapy, during therapy, and at the end of therapy. Most of the patients were treated with 2 to 4 courses of full-dose chemotherapy. The serum concentrations of VEGF were measured by a quantitative sandwich enzyme immunoassay using a commercial kit (Quantikine R, R \& D Systems Inc., Minneapolis, MN, USA). For each analysis, $100 \mu \mathrm{l}$ of serum was used. All analyses and calibrations were carried out in duplicate. Optical density was determined using a microtitre plate reader (Auto Reader, Sanko Junyaku, Tokyo, Japan) at $450 \mathrm{~nm}$.

We compared the S-VEGF concentrations between the responders and the nonresponders. The correlation between the S-VEGF concentrations and the various blood cells were analyzed. Data are expressed as the mean \pm standard deviation (S. D.) or standard error (S. E.), and were statistically analyzed using the Student's $t$ test, chi-squared test or Fisher's exact test; $p$ values $<0.05$ were considered statistically significant. All $p$ values are 2-tailed.

\section{RESULTS}

The pretreatment S-VEGF concentration of patients with lung cancer ranged from 64.3 to 1715.5 $\mathrm{pg} / \mathrm{ml}$ (mean, $378.3 \mathrm{pg} / \mathrm{ml}$ ). No association was found between the pretreatment S-VEGF concentration and age, gender, or clinical stage, but it did correlate with a ECOG performance status between PS1 and PS2 (Table 2). The mean pretreatment S-VEGF concentrations in the responders and nonresponders were $509.7 \mathrm{pg} / \mathrm{ml}$ and $382.8 \mathrm{pg} / \mathrm{ml}$, respectively. Although the S-VEGF concentrations in the responders were higher than those in the nonresponders, the difference was not significant. The serum concentration of VEGF in the responders decreased to a mean of $356.0 \mathrm{pg} / \mathrm{ml}$ during therapy and $304.1 \mathrm{pg} / \mathrm{ml}$ at the end of therapy. In contrast, the S-VEGF concen-

TABLE 1.

Clinical features of the responders and nonresponders to anticancer chemotherapy

\begin{tabular}{lcccc}
\hline Features & Responder & Non-responder & Total & $p$-values \\
\hline No of patients & 12 & 17 & 29 & \\
Age, mean \pm S.D. & $64.0 \pm 12.2$ & $63.5 \pm 9.7$ & $63.7 \pm 10.6$ & N.S. \\
Gender & 8 & & & \\
Male & 4 & 11 & 19 & N.S. \\
Female & 3 & 6 & 10 & \\
Histological type & 5 & 10 & & \\
Adenocarcinoma & 4 & 2 & 13 & N.S. \\
Squamous cell Ca & 3 & 5 & 7 & \\
Small cell Ca & 3 & 0 & 9 & N.S. \\
Clinical stages & 6 & 5 & 3 & \\
IIIa & & 12 & 8 & \\
IIIb & 7 & 11 & 18 & N.S. \\
IV & 3 & 1 & 18 & \\
Performance status & 2 & 5 & 4 & 7 \\
0 & & & & \\
1 & & & & \\
2 & & & & \\
\hline
\end{tabular}


TABLE 2.

Pretreatment serum VEGF concentration according to gender, histologic type, clinical stages and performance status

\begin{tabular}{lrcc}
\hline & Number & $\begin{array}{c}\text { VEGF level } \\
\text { mean } \pm \text { S.D. pg/ml }\end{array}$ & $p$-value \\
\hline Gender & & & \\
$\quad$ Male & 19 & $477.6 \pm 443.5$ & N.S. \\
Female & 10 & $355.0 \pm 201.8$ & \\
Histological type & & & \\
$\quad$ Adenocarcinoma & 13 & $453.1 \pm 275.1$ & \multirow{2}{*}{ N.S. } \\
Squamous cell Ca & 7 & $554.4 \pm 668.1$ & \\
Small cell Ca & 9 & $317.1 \pm 154.4$ & \\
Clinical stages & & & \\
IIIa & 3 & $109.3 \pm 48.0$ & \multirow{2}{*}{ N.S. } \\
IIIb & 6 & $543.2 \pm 591.7$ & \\
IV & 20 & $451.9 \pm 312.6$ & \\
Performance status & & & \\
0 & 18 & $345.3 \pm 224.4$ & \\
1 & 4 & $878.3 \pm 765.9$ & $<0.05$ \\
2 & 7 & $413.7 \pm 275.8$ & \\
\hline
\end{tabular}

VEGF: vascular endothelial growth factor

TABLE 3.

Correlation between the Serum-VEGF concentration and the WBC, neutrophil, lymphocyte, monocyte, and platelet counts, and the CRP concentration on admission

\begin{tabular}{lccccc}
\hline & WBC & Neutrophil & Monocyte & Platelet & CRP \\
\hline $\mathrm{R}^{*}$ & 0.668 & 0.716 & 0.692 & 0.476 & 0.600 \\
$p$ value & $<0.0001$ & $<0.0001$ & $<0.0001$ & $<0.01$ & $<0.0005$ \\
\hline
\end{tabular}

* R: Correlation coefficient

VEGF: vascular endothelial growth factor

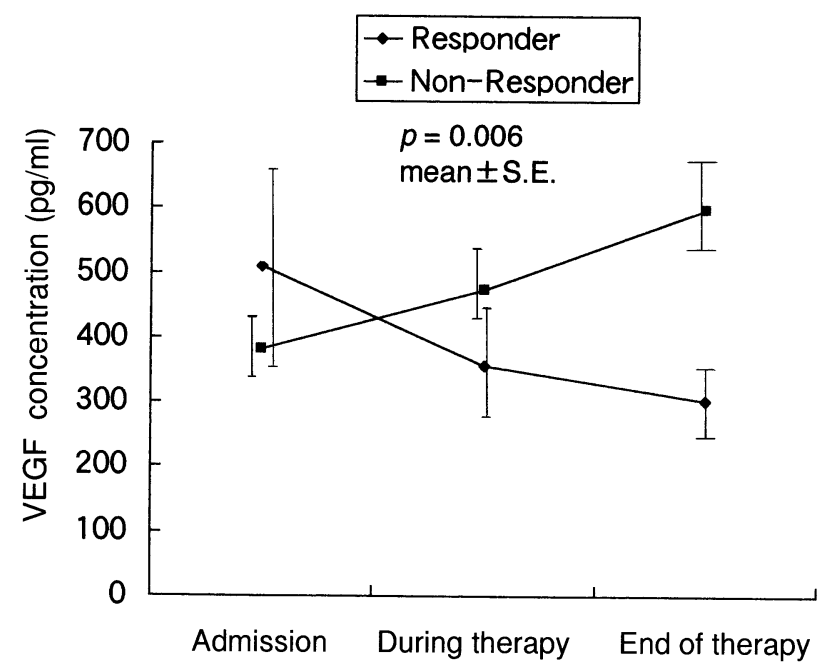

Fig. 1. Changing in the Serum-VEGF concentration during anticancer chemotherapy.

VEGF: vascular endothelial growth factor tration in the nonresponders increased to a mean of $474.2 \mathrm{pg} / \mathrm{ml}$ during therapy and $598.4 \mathrm{pg} / \mathrm{ml}$ at the end of therapy, both of which were significantly higher than those of the responders $(p=.006)$ (Fig. 1). The pretreatment S-VEGF concentration correlated with the WBC, the neutrophil count, the monocyte count, the platelet count, and the CRP (C-reactive protein) except lymphocyte count (Table 3 ).

\section{DISCUSSION}

Responders to anticancer chemotherapy generally have a better prognosis than do nonresponders. VEGF has been found to inhibit the maturation of dendritic cells, which are important antigen-presenting cells [3]. Immunosuppressive cytokines, such as transforming growth factor-beta (TGF- $B$ ), might suppress the host's anticancer immunity by stimulating VEGF production [4]. Therefore, the control of VEGF secretion could possibly be a key factor in inhibiting neoangiogenesis, reversing cancer-related immunosuppression and nullifying the promoting action of tumor growth factors on cancer dissemination. VEGF-mediated angiogenesis is considered to play an essential role in cancer progression and in the metastatic process [5]. In one study, the serum VEGF-positive rate was notably higher in stage III patients compared with stage I or stage II patients, and a significant difference was also observed between tumors less than $2 \mathrm{~cm}$ and tumors greater than $2 \mathrm{~cm}$ in diameter [6]. Ferrari et al. [7] reported that the S-VEGF concentrations in patients with advanced lung cancer were significantly higher than those with early-stage disease. However, Takigawa et al. [8] found that there was no difference between the S-VEGF concentration and the disease stage or presence of distant metastases in patients with lung cancer, leading them to conclude that the S-VEGF concentration might not be related to tumor burden. The pretreatment S-VEGF concentration in our patients in this study were not significantly different between the patients with stage IIIa, IIIb and IV lung cancer. Since our study did not include any patients with stage I or II lung cancer, we can not comment on Ferrari's findings. However, we believe that the results of this study does not support Takigawa's data, because the S-VEGF concentrations in responders decreased during therapy. Decreased S-VEGF concentration might reflect the dwindling tumor burden.

The relationship between the changes in the SVEGF concentration and response to anticancer 
chemotherapy has yet to be clarified. Lissoni et al. reported that there was no significant change in the mean S-VEGF concentration occurred during IL-2 therapy in patients with renal cell cancer. Moreover, the mean S-VEGF concentration did not change significantly during therapy in the patients who had a partial response (PR), stable disease (SD) or progressive disease (PD) [9]. It is well established that among patients who have lung cancer, responders to chemotherapy have a better prognosis than do nonresponders [10]. We found that the S-VEGF concentration in the responders decreased during therapy. On the other hand, the mean S-VEGF concentration in the nonresponders increased. We believe that these results are consistent with an improved survival for responders. Since the S-VEGF concentration decreased in the responders during chemotherapy, these results might suggest that this change improved neoangiogenesis and the immune response to the patient's cancer, thus leading to an improved quality of life and survival. Salven et al. [11] reported that a high concentration of S-VEGF was strongly associated with a poor response to therapy in patients with small cell lung cancer. On the other hand, Volm et al. [12] found that the expression of VEGF was lower in resistant lung tumors than in sensitive tumors. In the present no significant difference was observed in the pretreatment S-VEGF concentrations between the responders and nonresponders. Peripheral blood $\mathrm{T}$ lymphocytes and macrophages and lymphocytes infiltrating human cancers have been shown to express VEGF [13]. Hence, the S-VEGF may originate not only from the cancer cell, but also from tumor-infiltrating inflammatory cells and circulating peripheral blood cells $[14,15]$. Salven et al. [16] reported that a high blood VEGF concentration was associated with a high peripheral blood leukocyte count and platelet count, and the elevated S-VEGF in cancer patients was due to a rise of VEGF in blood cells. In this study, the pretreatment S-VEGF correlated with the leukocyte count, especially the neutrophil and the platelet count, consistent with the results of Salven's study.

Based upon the reduction of S-VEGF during anticancer chemotherapy, one might conclude that SVEGF may be a good marker for tumor burden and may help to monitor the responses of individual patients to anticancer therapy [17]. However, the SVEGF concentration can be influenced by various factors such as infection. We found in another study that the S-VEGF concentration was increased during infection in lung cancer patients. Therefore, it is unlikely that the S-VEGF concentration will prove to be a suitable tumor marker. We should use S-VEGF very carefully as a diagnostic and prognostic tool.

\section{CONCLUSIONS}

We found a significant change in the S-VEGF concentration in patients with lung cancer who responded well to anticancer chemotherapy. The SVEGF concentration may relate to tumor burden, however it can be influenced by various blood cells such as neutrophil which increases during infection. The reduction in the S-VEGF concentration that occurred in the responders during therapy may have a number of favorable effects, such as inhibiting neoangiogenesis and helping to facilitate the immune response in responders. Such effects may reasonably be expected to improve the patient's quality of life and prolong survival.

ACKNOWLEDGMENTS: The author is grateful to Professor Kotaro Oizumi and Associate Professor Toru Rikimaru, Department of Medicine, Kurume University School of Medicine, for their clinical advice and suggestions.

\section{REFERENCES}

1. Cohen T, Nahari D, Cerem LW, Neufeld G, and Levi BZ. Interleukin 6 induces the expression of vascular endothelial growth factor. J Biol Chem 1996; 271:736741.

2. Hanahan D, and Folkman J. Patterns and emerging mechanisms of the angiogenetic switch during tumorigenesis. Cell 1996; 86:353-364.

3. Gabrilovich DI, Chen HL, Girgis KR, Cunningham T, Meny GM et al. Production of vascular endothelial growth factor by human tumors inhibits the functional maturation of dendritic cells. Nat Med 1996; 2:10961103.

4. Rosenberg SA. The immunotherapy and gene therapy of cancer. J Clin Oncol 1992; 10:180-191.

5. Dvorak HF, Brown LF, Detmar M, and Dvorak AM. Vascular permeability factor/vascular endothelial growth factor, microvascular hyperpermeability, and angiogenesis. Amer J Pathol 1995; 146:1029-1039.

6. Yamamoto Y, Toi M, Kondo S, Matumoto T, Suzuki H et al. Concentrations of vascular endothelial growth factor in the sera of normal controls and cancer patients. Clin Cancer Res 1996; 2:821-826.

7. Ferrari G, and Scagliotti GV. Serum and urinary vascular endothelial growth factor levels in non-small cell lung cancer patients. Eur J Cancer 1996; 32A:2368-2369.

8. Takigawa N, Segawa Y, Fujimoto N, Hotta K, and Eguchi K. Elevated vascular endothelial growth factor levels in sera of patients with lung cancer. Anticancer Res 1998; 18:1251-1254. 
9. Lissoni P, Fumagalli L, Giani L, Rovelli F, Confalonieri $G$ et al. Vascular endothelial growth factor (VEGF) serum levels during cancer immunotherapy with IL-2: preliminary considerations. Int J Biol Marker 1998; 13:98-101.

10. Ganz PA, Figlin RA, and Haskell CM. Supportive care versus supportive care and combination chemotherapy in metastatic non-small cell lung cancer. Cancer 1989; 63:1271-1278.

11. Salven P, Ruotsalainen T, Mattson K, and Joensuu H. High pre-treatment serum level of vascular endothelial growth factor (VEGF) is associated with poor outcome in small-cell lung cancer. Int J Cancer 1998; 79:144-146.

12. Volm M, Koomagi R, Mattern J, and Stammler G. Angiogenic growth factors and their receptors in nonsmall cell lung carcinomas and their relationships to drug response in vitro. Anticancer Res 1997; 17:169-172.

13. Berse B, Brown LF, Van De Water L, Dvorak HF, and Senger DR. Vascular permeability factor (Vascular endothelial growth factor) gene is expressed differentially in normal tissues, macrophages, and tumors. Mol Biol Cell 1992; 3:211-220.

14. Freeman MR, Schneck FX, Gagnon ML, Corless C, Soker $\mathrm{S}$ et al. Peripheral blood $\mathrm{T}$ lymphocytes and lymphocytes infiltrating human cancers express vascular endothelial growth factor: A potential role for $\mathrm{T}$ cells in angiogenesis. Cancer Res 1995; 55:4140-4145.

15. Torisu H, Ono M, Kiryu H, Furue M, Ohmoto $Y$ et al. Macrophage infiltration correlates with tumor stage and angiogenesis in human malignant melanoma: possible involvement of $\mathrm{TNF} \alpha$ and IL- $1 \alpha$. Int J Cancer 2000; 85:182-188.

16. Salven P, Orpana A, and Joensuu H. Leucocytes and platelets of patients with cancer contain high level of vascular endothelial growth factor. Clin Cancer Res 1999; 5:487-491.

17. Benzakein EF, Ma MN, Brandt LR, Mentha G, Ruefenacht $\mathrm{D}$ et al. Elevated levels of angiogenic cytokines in the plasma of cancer patients. Int $\mathbf{J}$ Cancer $2000 ; 85: 40-45$. 